\title{
Morfologia de esporos de pteridófitas do Parque Estadual das Fontes do Ipiranga (São Paulo, SP, Brasil). Família: 10-Hymenophyllaceae
}

\author{
Kauê Fonseca ${ }^{1}$, Carolina Brandão Coelho ${ }^{1}$ e $\mathbb{D}$ Luciano Mauricio Esteves $^{1,2}$
}

Recebido: 19.03.2018; aceito: 8.08.2018

\begin{abstract}
Morphology of spores of pteridophyte from the Parque Estadual das Fontes do Ipiranga (São Paulo, São Paulo State, Brazil). Family: 10 - Hymenophyllaceae). Spores of pteridophyte species of the Family Hymenophyllaceae from the Parque Estadual das Fontes do Ipiranga were studied. The family is represented in the area by nine species: Abrodictyum rigidum (Sw.) Ebihara \& Dubuisson, Didymoglossum reptans (Sw.) C. Presl, Hymenophyllum caudiculatum Mart., Hymenophyllum hirsutum (L.) Sw., Hymenophyllum microcarpum Desv., Hymenophyllum polyanthos Sw., Polyphlebium angustatum (Carmich.) Ebihara \& Dubuisson, Trichomanes polypodioides Raddi and Vandenboschia radicans (Sw.) Copel. Descriptions, illustrations, and comments are provided for all the studied taxa.

Keywords: Abrodictyum, Didymoglossum, Hymenophyllaceae, Hymenophyllum, Polyphlebium, spores, Vandenboschia
\end{abstract}

RESUMO - (Morfologia de esporos de pteridófitas do Parque Estadual das Fontes do Ipiranga (São Paulo, SP, Brasil). Família: 10 - Hymenophyllaceae). Foram estudados os esporos de samambaias pertencentes à família Hymenophyllaceae do Parque Estadual das Fontes do Ipiranga. Esta família é representada na área por nove espécies: Abrodictyum rigidum (Sw.) Ebihara \& Dubuisson, Didymoglossum reptans (Sw.) C. Presl, Hymenophyllum caudiculatum Mart., Hymenophyllum hirsutum (L.) Sw., Hymenophyllum microcarpum Desv., Hymenophyllum polyanthos Sw., Polyphlebium angustatum (Carmich.) Ebihara \& Dubuisson, Trichomanes polypodioides Raddi e Vandenboschia radicans (Sw.) Copel. Para todos os táxons estudados são apresentadas descrições, ilustrações e observações.

Palavras-chave: Abrodictyum, Didymoglossum, esporos, Hymenophyllaceae, Hymenophyllum, Polyphlebium, Vandenboschia

\section{Introdução}

Os membros da família Hymenophyllaceae são facilmente distinguidos por suas lâminas foliares, geralmente com a espessura de uma única célula, e a monofilia da família não é questionada, embora a classificação intrafamiliar seja altamente controversa (Copeland 1938, Morton 1968, Iwatsuki 1977, Pichi-Sermolli 1977, Tryon \& Tryon 1982). Smith et al. (2006, 2008) e Iwatsuki (1990) reconhecem Hymenophyllaceae como uma família distinta, monofilética e basal no clado das samambaias leptosporangiadas, pertencendo a classe Polypodiopsida, ordem Hymenophyllales. É representada por nove gêneros: Abrodictyum C. Presl, Callistopteris Copel, Cephalomanes C. Presl, Crepidomanes C.Pres1, Didymoglossum Desv., Hymenophyllum Sm., Polyphlebium Copel., Vandenboschia Copel., Trichomanes L., e.; e cerca de
600 espécies, distribuídas principalmente em regiões tropicais e subtropicais (Ebihara et al. 2006, Smith et al. 2006). No Brasil, a família está representada por seis gêneros e 85 espécies (Windisch 2010, Hirai \& Prado 2011). Segundo Windisch (2010) o grupo ocorre principalmente na Mata Atlântica e Amazônia, como epífitas, terrestres ou rupícolas. Os soros são marginais, com indúsio cônico, tubular, ou bivalvado, e receptáculo alongado (nos Trichomanoides), abrigando esporos clorofilados, globosos e triletes (Smith et al. 2006).

No Parque Estadual das Fontes do Ipiranga (PEFI) foram registrados seis gêneros e nove espécies: Abrodictyum (A. rigidum (Sw.) Ebihara \& Dubuisson), Hymenophyllum (H. caudiculatum Mart., H. hirsutum (L.) Sw., H. microcarpum Desv., H. polyanthos Sw.), Didymoglossum (D. reptans (Sw.) C. Presl), Polyphlebium (P. angustatum (Carmich.) Ebihara \& Dubuisson), Trichomanes (T. polypodioides Raddi)

1. Instituto de Botânica, Avenida Miguel Estéfano, 3687, 04301-902 São Paulo, SP, Brasil

2. Autor para correspondência: lesteves2001@yahoo.com.br 
e Vandenboschia (V. radicans (Sw.) Copel) (Hirai \& Prado 2011).

Estudos sobre a morfologia dos esporos das espécies de Hymenophyllaceae são escassos. Dentre os principais autores temos Lugardon (1981), Morbelli (1983), Morbelli et al. (2010), Tryon \& Lugardon (1991), Tryon \& Tryon (1982), entretanto, a análise palinológica é baseada em microscopia eletrônica de varredura e de transmissão.

Análise morfológica dos esporos das espécies de Hymenophyllaceae presentes no Parque Estadual das Fontes do Ipiranga complementa os estudos taxonômicos da família e amplia o material de referência para estudos de precipitação esporopolínica e a palinologia de sedimentos na área.

\section{Materiais e métodos}

O planejamento do estudo da presente flora e os dados referentes ao PEFI encontram-se descritos no trabalho de Melhem et al. (1981). A chave para identificação da família Hymenophyllaceae foi publicada por Prado (2004). A classificação segue a circunscrição para a família conforme apresentada em Ebihara et al. (2006).

O material foi coletado a partir de exsicatas depositadas no Herbário Científico do Estado "Maria Eneyda P. Kauffmann Fidalgo" (SP). Quando os espécimes se apresentaram inférteis, ou com quantidade insuficiente, optou-se por coletas a partir de exsicatas provenientes de outras localidades.

Os esporos foram preparados segundo a técnica de acetólise (Erdtman 1960) e acetólise láctica (Raynal, J. \& Raynal, A.) quando se mostrou necessário devido à fragilidade do material. As ilustrações foram obtidas utilizando-se fotomicroscópio Olympus BX 50 acoplado a uma câmara de vídeo e microcomputador (PC) e o programa Pro-Plus versão 3 para Windows. A partir de um microscópio optico foram medidos os eixos polar (P) e equatorial maior (EM), o comprimento da lesão (L), a margem da lesão (ML) e a esporoderme (E), determinando-se a média, o intervalo de confiança a $95 \%$ e o coeficiente de variabilidade da amostra. Para cada parâmetro foram tomadas 25 medidas, sempre que possível. A nomenclatura utilizada nas descrições dos esporos foi baseada em Lellinger (2002). As lâminas preparadas encontram-se depositadas na Palinoteca do Núcleo de Pesquisa em Palinologia do Instituto de Botânica/São Paulo.

\section{Resultados e Discussão}

Família Hymenophyllaceae

Abrodictyum C. Presl

Abrodictyum rigidum (Sw.) Ebihara e Dubuisson

Figuras 1-5

Forma: trilete, esferoidal.

Lesão: braço da lesão aproximadamente $4 / 5$ do raio com margem evidente.

Estrutura: exosporo papilado, formado de duas camadas, a mais externa aproximadamente duas vezes mais espessa do que a interna. Perisporo tênue, psilado.

Medidas $(\mu \mathrm{m}): \mathrm{P}=32,9 \pm 0,6 ; \mathrm{EM}=48,0 \pm 0,5$; $\mathrm{L}=22,0 \pm 0,7 ; \mathrm{ML}=1,6 \pm 0,1 ; \mathrm{E}=2,4 \pm 0,1$.

Observação: o material 1-X-1936, O. Handro s.n. (SP43039), citado por Prado (2011), não foi estudado por apresentar esporos em quantidade insuficiente para a análise.

Material examinado: 9-IV-1976, J.A. Corrêa 130 (SP155121).

\section{Didymoglossum Desv.}

\section{Didymoglossum reptans (Sw.) C. Presl}

Figuras 6-9

Forma: trilete, esferoidal.

Lesão: braço da lesão aproximadamente $3 / 4$ do raio com margem tênue.

Estrutura: exosporo papilado, formado de duas camadas de mesma espessura. Perisporo psilado e tênue.

Medidas $(\mu \mathrm{m}): \mathrm{EM}=54,4 \pm 4,3 ; \mathrm{L}=16,2 \pm 1,8 ;$ $\mathrm{ML}=1,3 \pm 0,1 ; \mathrm{E}=1,1 \pm 0,1$.

Observações: para o eixo polar $(\mathrm{P})$ não houve medidas suficiente para a análise estatística. Foi considerada somente o eixo equatorial (EM), lesão (L), margem da lesão (ML) e esporoderme (E).

Material examinado: 24-IV-1951, O. Handro 246 (SP39245).

\section{Hymenophyllum Sm.}

\section{Hymenophyllum caudiculatum Mart.}

Figuras 10-13 
Forma: trilete, esferoidal.

Lesão: braço da lesão se estendendo por todo o raio, com margem evidente.

Estrutura: exosporo papilado "verrugoso irregular", de duas camadas de mesma espessura. Perisporo psilado e tênue.

Medidas $(\mu \mathrm{m}): \mathrm{P}=45,2 \pm 1,7 ; \mathrm{EM}=66,0 \pm 2,2$; $\mathrm{L}=32,3 \pm 1,5 ; \mathrm{ML}=1,9 \pm 0,11 ; \mathrm{E}=2,4 \pm 0,1$.

Observação: o material 24-VII-1933, W. Hoehne 278 (SPF), citado por Prado \& Hirai (2011), não foi estudado por apresentar esporos em quantidade insuficiente para a análise.

Material examinado: 10-X-1940, O. Handro s.n. (SP44142).

Hymenophyllum hirsutum (L.) Sw.

Figuras 14-17

Forma: trilete, esferoidal.

Lesão: braço da lesão se estendendo por todo o raio, com margem evidente.

Estrutura: exosporo papilado, de duas camadas de mesma espessura. Perisporo tênue e psilado.

Medidas $(\mu \mathrm{m}): \mathrm{P}=43,8 \pm 3,1 ; \mathrm{EM}=53,1 \pm 1,2$; $\mathrm{L}=24,4 \pm 0,6 ; \mathrm{ML}=2,3 \pm 0,1 ; \mathrm{E}=2,2 \pm 0,1$.

Material examinado: 19-IX-1938, O. Handro s.n. (SP52527).

\section{Hymenophyllum microcarpum Desv.}

Figuras 18-19, 20-22

Forma: trilete, esferoidal.

Lesão: braço da lesão se estendendo por todo o raio, com margem evidente.

Estrutura: exosporo equinado, formado de duas camadas, sendo a camada externa aproximadamente duas vezes mais espessa que a interna. Perisporo psilado e tênue.

Medidas $(\mu \mathrm{m}): \mathrm{P}=40,4 \pm 0,5 ; \mathrm{EM}=55,5 \pm 0,6$; $\mathrm{L}=25,7 \pm 0,5 ; \mathrm{ML}=2,2 \pm 0,1 ; \mathrm{E}=2,4 \pm 0,1$.

Material examinado: 24-VII-1933, W. Hoehne 278 (SPF).

\section{Hymenophyllum polyanthos Bosch.}

Figuras 23-27

Forma: trilete, esferoidal.
Lesão: braço da lesão se estendendo por todo o raio, com margem evidente.

Estrutura: Exosporo papilado a gemulado, formado de duas camadas, sendo a camada externa aproximadamente duas vezes mais espessa que a interna. Perisporo psilado e tênue.

Medidas $(\mu \mathrm{m}): \mathrm{P}=56,6 \pm 1,0 ; \mathrm{EM}=72,4 \pm 0,7$; $\mathrm{L}=35,9 \pm 0,8 ; \mathrm{ML}=2,7 \pm 0,1 ; \mathrm{E}=3,6 \pm 0,2$.

Observação: o material 30-IV-1974, J.A. Corrêa 49 (SP), citado por Prado \& Hirai (2011), não foi estudado por apresentar esporos em quantidade insuficiente para a análise.

Material examinado: 21-III-1996, J.Prado 786 (SP313553).

Polyphlebium Copel.

Polyphlebium angustatum (Carmich.) Ebihara \& Dubuisson.

Figuras 28-31

Forma: trilete, esferoidal.

Lesão: braço da lesão se estendendo por todo o raio, com margem evidente.

Estrutura: Exosporo papilado, formado de duas camadas de mesma espessura. Perisporo psilado e tênue.

Medidas $(\mu \mathrm{m}): \mathrm{P}=25,7 \pm 0,4 ; \mathrm{EM}=33,5 \pm 0,5$; $\mathrm{L}=13,0 \pm 0,2 ; \mathrm{ML}=1,5 \pm 0,1 ; \mathrm{E}=1,4 \pm 0,1$.

Observação: os materiais 26-IV-1951, O. Handro 247 (SP) e G. Eiten et al. 2050 (SP), citados por Prado \& Hirai (2011), não foram estudados por apresentarem esporos em quantidade insuficiente para a análise.

Material examinado: 1-VIII-2008, J.Prado 2004 (SP448114).

\section{Trychomanes L.}

\section{Trichomanes polypodioides L.}

Figuras 32-35

Forma: trilete, esferoidal.

Lesão: braço da lesão se estendendo por todo o raio, com margem de difícil visualização.

Estrutura: exosporo papilado, formado de duas camadas de mesma espessura. Perisporo psilado e tênue. 

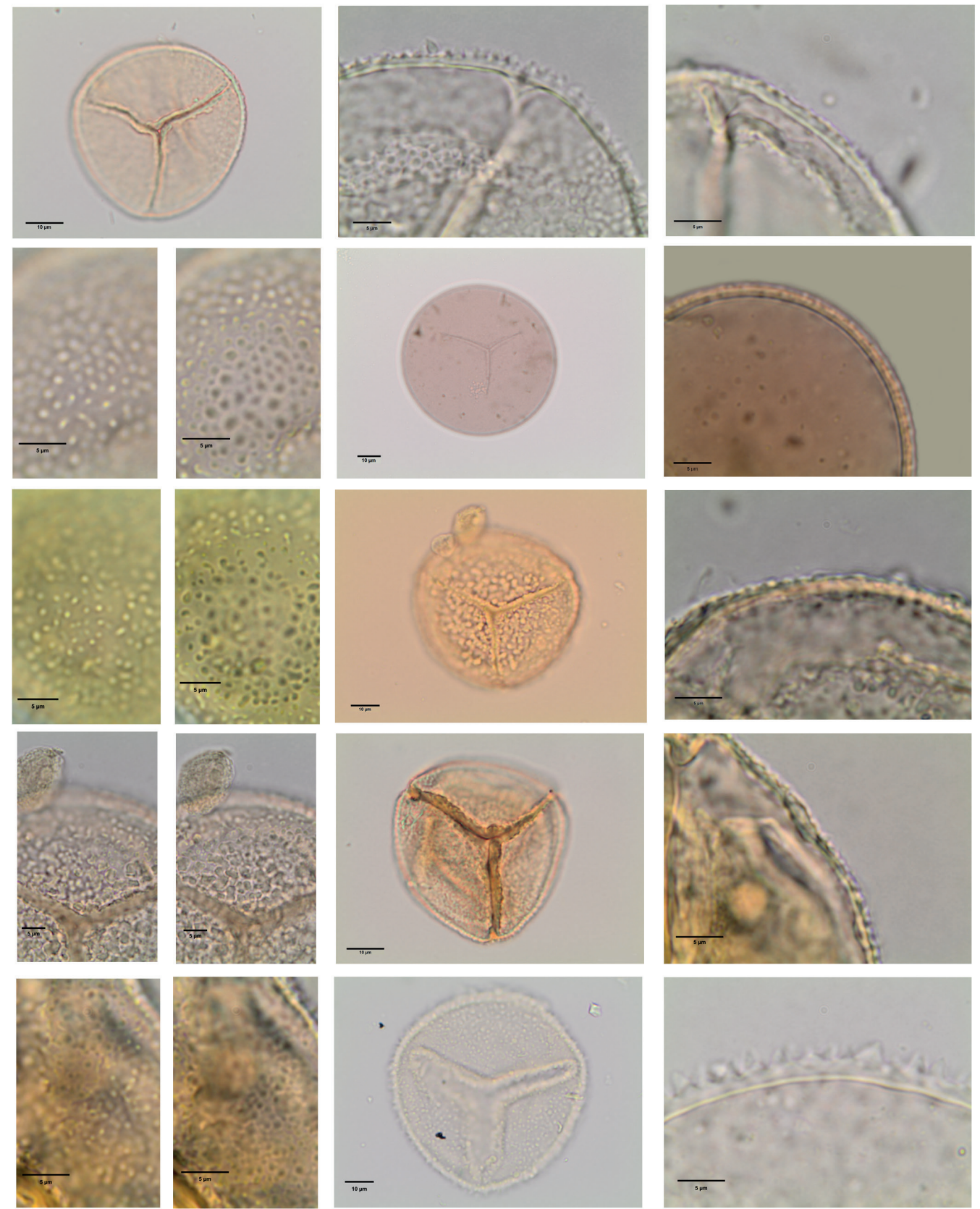

Figuras 1-19. Fotomicrografias dos esporos dos gêneros Abrodictyum C. Presl, Didymoglossum Desv. e Hymenophyllum Sm. 1-5. Abrodyctium regidum. (Sw.) Ebihara \& Dubuisson. 1. Vista polar proximal. 2. Corte óptico da esporoderme, exosporo papilado. 3. Corte óptico, esporoderme de duas camadas. 4-5. Análise de L.O. 6-9. Didymoglossum reptans (Sw.) C. Presl 6. Vista polar proximal.7. Corte óptico, detalhe da esporoderme. 8-9. Análise de L.O. 10-13. Hymenophyllum caudiculatum Mart. 10. Vista polar.proximal. 11. Corte óptico da esporoderme. 12-13. Análise de L.O. 14-17. Hymenophyllum hirsutum (L.) Sw. 14. Vista polar proximal. 15. Corte óptico da esporoderme. 16-17. Análise de L.O. 18-19. Hymenophyllum microcarpum Desv. 18. Vista polar proximal. 19. Corte óptico da esporoderme. Escala: 2-5,7-9,11-13,15-17,19=5 $\mu \mathrm{m} ; 1,6,10,14,18=10 \mu \mathrm{m}$.

Figures 1-19. Photomicrographs of spores of the genus Abrodictyum C. Pres1, Didymoglossum Desv, and Hymenophyllum Sm. 1-5. Abrodyctium regidum. (Sw.) Ebihara \& Dubuisson.

1. Proximal polar view. 2. Optical section of the sporoderm, exospore papillate. 3. Optical section, two-layer sporoderm. 4-5. L.O. analysis .6-9. Didymoglossum reptans (Sw.) C. Presl 6. Proximal polar view. 7. Optical section, detail of the sporoderm. 8-9. L.O. analysis. 10-13. Hymenophyllum caudiculatum Mart. 10. Polar view. 11. Optical section of the sporoderm. 12-13. L.O. analysis. 14-17. Hymenophyllum hirsutum (L.) Sw. 14. Proximal polar view. 15. Optical section of the sporoderm. 16-17. L.O. analysis. 18-19. Hymenophyllum microcarpum Desv. 18. Proximal polar view. 19. Optical section of the sporoderm. Scale bars: 2-5, 7-9, 11-13, 15-17, $19=5 \mu \mathrm{m} ; 1,6,10,14,18=10 \mu \mathrm{m}$. 

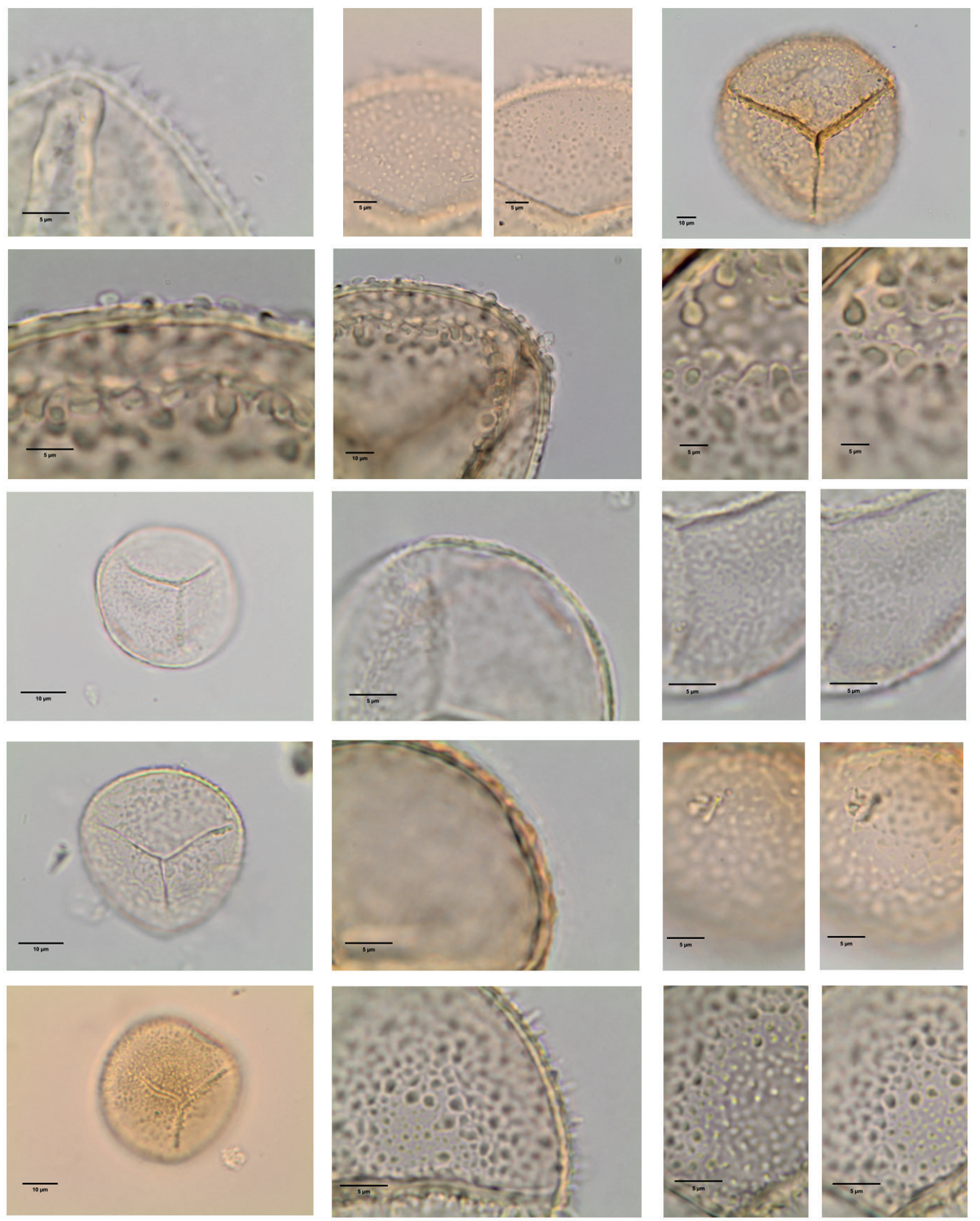

Figuras 20-39. Fotomicrografias dos esporos dos gêneros Hymenophyllum Sm., Polyphlebium Copel., Trichomanes L. e Vandenboschia Copel. 20-22. Hymenophyllum microcarpum Desv. 20. Corte óptico da esporoderme, exosporo aculeado. 21-22. Análise de L.O. 23-27. Hymenophyllum polyanthos Sw. 23. Vista polar proximal. 24. Corte óptico da esporoderme, duas camadas. 25. Corte óptico, exosporo papilado a gemulado. 26-27. Análise de L.O. 28-31. Polyphlebium angustatum (Carmich.) Ebihara \& Dubuisson. 28. Vista polar proximal. 29. Corte óptico da esporoderme. 30-31. Análise de L.O. 32-35. Trichomanes polypodioides Raddi. 32. Vista polar.proximal. 33. Corte óptico da esporoderme. 34-35. Análise de L.O. 36-39. Vandenboschia radicans (Sw.) Copel. 36. Vista polar proximal. 37. Corte óptico, exosporo equinado. 38-39. Análise de L.O. Escala: 20-22, 24-27, 29-31, 33-35, 37-39=5 $\mu \mathrm{m} ; 23,28,32,36=10 \mu \mathrm{m}$.

Figures 20-39. Photomicrographs of spores of the genus Hymenophyllum Sm., Polyphlebium Copel., Trichomanes L. and Vandenboschia Copel. 20-22. Hymenophyllum microcarpum Desv. 20. Optical section of the sporoderm, exposore aculeate. 21-22. L.O. analysis. 23-27. Hymenophyllum polyanthos Sw. 23. Proximal polar view. 24. Optical section of the sporoderm, two-layers. 25. Optical section, exospore papillate to gemmulate. 26-27. L.O. analysis. 28-31. Polyphlebium angustatum (Carmich.) Ebihara \& Dubuisson. 28. Proximal polar view. 29. Optical section of the sporoderm. 30-31. L.O. analysis. 32-35. Trichomanes polypodioides Raddi. 32. Polar view. 33. Optical section of the sporoderm. 34-35. L.O. analysis. 36-39. Vandenboschia radicans (Sw.) Copel. 36. Proximal polar view. 37. Optical section, exospore echinate. 38-39. L.O. analysis. Scale bars: 20-22, 24-27, 29-31, 33-35, 37-39= $5 \mu \mathrm{m} ; 23,28,32,36=10 \mu \mathrm{m}$. 
Medidas $(\mu \mathrm{m}): \mathrm{P}=29,3 \pm 1,1 ; \mathrm{EM}=38,4 \pm 0,5 ; \mathrm{L}=$ $18,4 \pm 0,5 ; \mathrm{ML}=1,2 \pm 0,1 ; \mathrm{E}=1,8 \pm 0,1$.

Observações: os materiais 2-IX-1976, P.H. Davis et al. 60427 (UEC); 20-X-2003, J. Prado \& D.M. Vital 1452 (SP), 5-II-2004, J. Prado 1456 (SP); citados por Prado \& Hirai (2011), não foram estudados por apresentarem esporos em quantidade insuficiente para a análise.

Material examinado: 30-VI-1936, O. Handro s.n. (SP44450).

\section{Vandenboschia Copel.}

Vandenboschia radicans (Sw.) Copel.

Figuras 36-39

Forma: trilete, esferoidal.
Lesão: braço da lesão se estendendo por todo o raio, com margem de difícil visualização.

Estrutura: exosporo equinado, formado de duas camadas de mesma espessura. Perisporo psilado e tênue.

Medidas $(\mu \mathrm{m}): \mathrm{P}=34,5 \pm 0,8 ; \mathrm{EM}=46,0 \pm 0,9$; $\mathrm{L}=18,3 \pm 0,9 ; \mathrm{ML}=1,4 \pm 0,1 ; \mathrm{E}=1,7 \pm 0,1$.

Observação: o material 20-X-1936, O. Handro s.n. (SP44449), citado por Prado \& Hirai (2011), não foi estudado por apresentar esporos em quantidade insuficiente para a análise.

Material examinado: 20-X-1936, O. Handro s.n (SPF64911).

Chave para as espécies de Hymenophyllaceae

1. Ornamentação do exosporo papilada

1. Ornamentação do exosporo não papilada

2. Ornamentação do exósporo aculeada

2. Ornamentação do exosporo equinado Hymenophyllum microcarpum 3. Ø E.M. $<35 \mu \mathrm{m}$

3. $\varnothing$ E.M. $>35 \mu \mathrm{m}$ .. Vandenboschia radicans

4. Presença de glóbulos no exosporo Polyphlebium angustatum

4. Ausência de glóbulos no exosporo

5. Lesão $3 / 4$ do raio Hymenophyllum polyanthos

5. Lesão $>3 / 4$ do raio

6. Papilas fundidas Hymenophyllum caudiculatum

6. Papilas não-fundidas Abrodictyum rigidum

Trichomanes polypodioides Hymenophylum hirsutum

\section{Literatura citada}

Copeland, E.B. 1938. Genera Hymenophyllacearum. Philippine Journal of Science 51: 2-110.

Ebihara, A., Dubuisson, J.-Y., Iwatsuki, K., Hennequin, S. \& Ito, M. 2006. A Taxonomic revision of Hymenophyllaceae. Blumea 51: 221-280.

Erdtman, G. 1960. The acetolysis method. A revised description. Svensk Botanisk Tidskrift 54: 561-564.

Hirai, R.Y. \& Prado, J. 2011. Criptógamos do Parque Estadual das Fontes do Ipiranga, São Paulo, SP, Brasil. Pteridophyta: 10. Hymenophyllaceae. Hoehnea 38: 501-510.

Iwatsuki, K. 1977. Studies in the systematics of filmy ferns III. An observation on the involucres. Botanical Magazine 90: 259-267.

Iwatsuki, K. 1990. Hymenophyllaceae. In: K.U. Kramer \& P.S. Green (eds.). The families and genera of vascular plants, v. 1, Pteridophytes and Gymnosperms. SpringerVerlag, Berlim, pp. 157-163.
Lellinger, D.B. 2002. A modern multilingual glossary for Taxonomic Pteridology. Pteridologia n. 3. American Fern Society, Washington.

Melhem, T.S., Giulietti, M., Forero, E., Barroso, G.M., Silvestre, M.S.F., Jung, S.L., Makino, H., Fiuza de Melo, M.M.R., Chiea, S.C., Wanderley, M.G.L., Kirizawa, M. \& Muniz, C. 1981. Planejamento para a elaboração da "Flora Fanerogâmica da Reserva do Parque Estadual das Fontes do Ipiranga (São Paulo, Brasil)". Hoehnea 9: 63-74.

Morbelli, M. A. 1983. Estudio de esporas de Pteridofitas del Noroeste de Argentina. Hymenophyllaceae. In: Org. Comm. (ed.). V Simp. Argentino Paleobot. \& Palinol., Buenos Aires, pp. 36.

Morbelli, M.A., Piñeiro, M.R., Giudice, G.E. Spore morphology and wall ultrastructure of Hymenophyllaceae Link (Pteridophyta) from north-west Argentina. Grana 49: 37-46. 
Morton, C.V. 1968. The genera, subgenera, and sections of the Hymenophyllaceae. Contributions from the United States National Herbarium 38: 153-214.

Pichi-Sermolli, R.E.G. 1977. Tentamen pteridophytorum genera in taxonomicum ordinem redigendi. Webbia 31: 315-512.

Prado, J. 2004. Criptógamos do Parque Estadual das Fontes do Ipiranga, São Paulo, SP. Pteridophyta: chave para as famílias; 2. Blechnaceae. Hoehnea 31: 1-10

Raynal, J. \& Raynal, A. 1971. Une techinique de preparation des grains de pollen fragiles. Adansonia 11: 77-79.

Smith, A.R., Pryer, K.M., Schuettpelz, E., Korall, P., Schneider, H. \& Wolf, P.G. 2006. A classification for extant ferns. Taxon 55: 705-731.

Smith, A.R., Pryer, K.M., Schuettpelz, E., Korall, P., Schneider, H. \& Wolf, P.G. 2008. Fern classification. In: T.A. Ranker \& C.H. Haufler (eds.). Biology and evolution of ferns and lycophytes. Cambridge University Press, Cambridge, pp. 417-467.
Tryon, R.M. \& Tryon, A.F. 1982. Ferns and allied plants, with special reference to tropical America. SpringerVerlag, New York.

Tryon, A.F. \& Lugardon, B. 1991. Spores of the Pteridophyta: Surface, wall structure and diversity based on electron microscope studies. Springer, New York.

Windisch, P.G. 2010. Hymenophyllaceae. In: R.C. Forzza, J.F. Baumgratz, C.E.M, Bicudo, D. Canhos, A.A. Carvalho Jr., A. Costa, D.P. Costa, M. Hopkins, M.P. Leitman, L.G. Lohmann, E.N. Lughadha, L.C. Maia, G. Martinelli, M. Menezes, M.P. Morin, M. Nadruz, A.L. Peixoto, J.R. Pirani, J. Prado, L.P. Queiroz, S. de Souza, V.C. Souza, J.R. Stehmann, L.S. Sylvestre, B.M.T. Walter \& D.C. Zappi (eds.). Catálogo de Plantas e Fungos do Brasil. Jardim Botânico do Rio de Janeiro, Rio de Janeiro, v. 1, pp. 538-540. 